\title{
Some Endpoint Results for $\beta$-Generalized Weak Contractive Multifunctions
}

\author{
H. Alikhani, ${ }^{1}$ D. Gopal, ${ }^{2}$ M. A. Miandaragh, ${ }^{1}$ Sh. Rezapour, ${ }^{1}$ and N. Shahzad ${ }^{3}$ \\ ${ }^{1}$ Department of Mathematics, Azarbaijan University of Shahid Madani, Azarshahr, Tabriz, Iran \\ ${ }^{2}$ Department of Applied Mathematics and Humanities, S. V. National Institute of Technology, \\ Surat, Gujarat 395007, India \\ ${ }^{3}$ Department of Mathematics, King Abdulaziz University, P.O. Box 80203, Jeddah 21859, Saudi Arabia
}

Correspondence should be addressed to N. Shahzad; nshahzad@kau.edu.sa

Received 3 August 2013; Accepted 29 August 2013

Academic Editors: M. M. Cavalcanti and N. Herisanu

Copyright (C) 2013 H. Alikhani et al. This is an open access article distributed under the Creative Commons Attribution License, which permits unrestricted use, distribution, and reproduction in any medium, provided the original work is properly cited.

We introduce $\beta$-generalized weak contractive multifunctions and give some results about endpoints of the multifunctions. Also, we give some results about role of a point in the existence of endpoints.

\section{Introduction}

Let $(X, d)$ be a metric space, $C B(X)$ the collection of all nonempty bounded and closed subsets of $X$, and $H$ the Hausdorff metric with respect to $d$; that is, $H(A, B)=$ $\max \left\{\sup _{x \in A} d(x, B), \sup _{y \in B} d(y, A)\right\}$ for all $A, B \in C B(X)$, where $d(x, B)=\inf _{y \in B} d(x, y)$. Let $T: X \rightarrow 2^{X}$ be a multifunction. An element $x \in X$ is said to be a fixed point of $T$ whenever $x \in T x$. Also, an element $x \in X$ is said to be an endpoint of $T$ whenever $T x=\{x\}$ [1]. We say that $T$ has the approximate endpoint property whenever $\inf _{x \in X} \sup _{y \in T x} d(x, y)=0$ [1]. Let $f: X \rightarrow X$ be a mapping. We say that $f$ has the approximate endpoint property whenever inf $\operatorname{inX}_{d} d(x, f x)=0$ [1]. Also, the function $g: \mathbb{R} \rightarrow \mathbb{R}$ is called upper semicontinuous whenever $\limsup _{n \rightarrow \infty} g\left(\lambda_{n}\right) \leq g(\lambda)$ for all sequences $\left\{\lambda_{n}\right\}_{n \geq 1}$ with $\lambda_{n} \rightarrow \lambda$ [2]. In 2010, Amini-Harandi defined the concept of approximate endpoint property for multifunctions and proved the following result (see [1]).

Theorem 1. Let $\psi:[0, \infty) \rightarrow[0, \infty)$ be an upper semicontinuous function such that $\psi(t)<t$ and $\liminf _{t \rightarrow \infty}(t-\psi(t))>0$ for all $t>0,(X, d)$ a complete metric space, and $T: X \rightarrow$ $C B(X)$ a multifunction satisfing $H(T x, T y) \leq \psi(d(x, y))$ for all $x, y \in X$. Then $T$ has a unique endpoint if and only if T has the approximate endpoint property.
Then Moradi and Khojasteh introduced the concept of generalized weak contractive multifunctions and improved Theorem 1 by providing the following result [3].

Theorem 2. Let $\psi:[0, \infty) \rightarrow[0, \infty)$ be an upper semicontinuous function such that $\psi(t)<t$ and $\liminf _{t \rightarrow \infty}(t-$ $\psi(t))>0$ for all $t>0,(X, d)$ a complete metric space, and $T$ : $X \rightarrow C B(X)$ a generalized weak contractive multifunction; that is, $T$ satisfies $H(T x, T y) \leq \psi(N(x, y))$ for all $x, y \in X$, where $N(x, y)=\max \{d(x, y), d(x, T x), d(y, T y),(d(x, T y)+$ $d(y, T x)) / 2\}$. Then $T$ has a unique endpoint if and only if $T$ has the approximate endpoint property.

In this paper, we introduce $\beta$-generalized weak contractive multifunctions, and by adding some conditions to assumptions of the results, we give some results about endpoints of $\beta$-generalized weak contractive multifunctions. In 2012, the technique of $\alpha$ - $\psi$-contractive mappings was introduced by Samet et al. [4]. Later, some authors used it for some subjects in fixed point theory (see for example [5$8]$ ) or generalized it by using the method of $\beta-\psi$-contractive multifunctions (see e.g., [9-12]).

Let $(X, d)$ be a metric space and $\beta: 2^{X} \times 2^{X} \rightarrow$ $[0, \infty)$ a mapping. A multifunction $T: X \rightarrow 2^{X}$ is called $\beta$-generalized weak contraction whenever there 
exists a nondecreasing, upper, semicontinuous function $\psi$ : $[0,+\infty) \rightarrow[0,+\infty)$ such that $\psi(t)<t$ for all $t>0$ and

$$
\beta(T x, T y) H(T x, T y) \leq \psi(N(x, y))
$$

for all $x, y \in X$. We say that $T$ is $\beta$-admissible whenever $\beta(A, B) \geq 1$ implies that $\beta(T x, T y) \geq 1$ for all $x \in A$, and $y \in B$, where $A$ and $B$ are subsets of $X$. We say that $T$ has the property $(R)$ whenever for each convergent sequence $\left\{x_{n}\right\}$ in $X$ with $x_{n} \rightarrow x$ and $\beta\left(T x_{n-1}, T x_{n}\right) \geq 1$ for all $n \geq 1$, we have $\beta\left(T x_{n}, T x\right) \geq 1$. One can find idea of the property $(R)$ for mappings in [13]. We say that $T$ has the property $(K)$ whenever for each sequence $\left\{x_{n}\right\}$ in $X$ with $\beta\left(T x_{n-1}, T x_{n}\right) \geq 1$ for all $n \geq 1$, there exists a natural number $k$ such that $\beta\left(T x_{m}, T x_{n}\right) \geq 1$ for all $m>n \geq k$. Finally, we say that $T$ has the property $(H)$ whenever for each $\varepsilon>0$, there exists $z \in X$ such that $\sup _{a \in T z} d(z, a)<\varepsilon$ implies that for every $x \in X$ there exists $y \in T x$ such that $H(T x, T y)=\sup _{b \in T y} d(y, b)$. A multifunction $T: X \rightarrow 2^{X}$ is called lower semicontinuous at $x_{0} \in X$ whenever for each sequence $\left\{x_{n}\right\}$ in $X$ with $x_{n} \rightarrow x_{0}$ and every $y \in T x_{0}$, there exists a sequence $\left\{y_{n}\right\}$ in $X$ with $y_{n} \in T x_{n}$ for all $n \geq 1$ such that $y_{n} \rightarrow y[14]$.

\section{Main Results}

Now, we are ready to state and prove our main results.

Theorem 3. Let $(X, d)$ be a complete metric space, $\beta: 2^{X} \times$ $2^{X} \rightarrow[0, \infty)$ a mapping, and $T: X \rightarrow C B(X) a$ $\beta$-admissible, $\beta$-generalized weak contractive multifunction which has the properties $(R),(K)$, and $(H)$. Suppose that there exist a subset $A$ of $X$ and $x_{0} \in A$ such that $\beta\left(A, T x_{0}\right) \geq 1$. Then $T$ has an endpoint if and only if $T$ has the approximate endpoint property.

Proof. It is clear that if $T$ has an endpoint, then $T$ has the approximate endpoint property. Conversely, suppose that $T$ has the approximate endpoint property. Choose $A \subset X$ and $x_{0} \in A$ such that $\beta\left(A, T x_{0}\right) \geq 1$. Since $T$ has the approximate endpoint property, for each $\varepsilon>0$, there exists $z \in X$ such that $\sup _{a \in T z} d(z, a)<\varepsilon$. Now by using the condition $(H)$, choose $x_{1} \in T x_{0}$ such that $H\left(T x_{0}, T x_{1}\right)=\sup _{a \in T x_{1}} d\left(x_{1}, a\right)$. Also, choose $x_{2} \in T x_{1}$ such that $H\left(T x_{1}, T x_{2}\right)=\sup _{a \in T x_{2}} d\left(x_{2}, a\right)$, and by continuing this process, we find a sequence $\left\{x_{n}\right\}$ in $X$ such that $x_{n} \in T x_{n-1}$ and

$$
H\left(T x_{n-1}, T x_{n}\right)=\sup _{a \in T x_{n}} d\left(x_{n}, a\right)
$$

for all $n \geq 1$. Since $\beta\left(A, T x_{0}\right) \geq 1$ and $T$ is $\beta$-admissible, $\beta\left(T x_{0}, T x_{1}\right) \geq 1$. By using induction, it is easy to see that $\beta\left(T x_{n-1}, T x_{n}\right) \geq 1$ for all $n \geq 1$. Thus, we obtain

$$
\begin{aligned}
d\left(x_{n}, x_{n+1}\right) & \leq \sup _{a \in T x_{n}} d\left(x_{n}, a\right)=H\left(T x_{n-1}, T x_{n}\right) \\
& \leq \beta\left(T x_{n-1}, T x_{n}\right) H\left(T x_{n-1}, T x_{n}\right) \\
& \leq \psi\left(N\left(x_{n-1}, x_{n}\right)\right)
\end{aligned}
$$

for all $n \geq 1$. If $N\left(x_{n-1}, x_{n}\right)=d\left(x_{n-1}, x_{n}\right)$, then

$$
d\left(x_{n}, x_{n+1}\right) \leq \psi\left(d\left(x_{n-1}, x_{n}\right)\right)
$$

If $N\left(x_{n-1}, x_{n}\right)=d\left(x_{n-1}, T x_{n-1}\right)$, then

$$
d\left(x_{n}, x_{n+1}\right) \leq \psi\left(d\left(x_{n-1}, T x_{n-1}\right)\right) \leq \psi\left(d\left(x_{n-1}, x_{n}\right)\right) .
$$

If $N\left(x_{n-1}, x_{n}\right)=d\left(x_{n}, T x_{n}\right)$, then

$$
d\left(x_{n}, x_{n+1}\right) \leq \psi\left(d\left(x_{n}, T x_{n}\right)\right) \leq \psi\left(d\left(x_{n}, x_{n+1}\right)\right),
$$

and so $d\left(x_{n}, x_{n+1}\right)=0$. Thus, $d\left(x_{n}, x_{n+1}\right) \leq \psi\left(d\left(x_{n-1}, x_{n}\right)\right)$. If

$$
\begin{aligned}
N\left(x_{n-1}, x_{n}\right) & =\frac{d\left(x_{n}, T x_{n-1}\right)+d\left(x_{n-1}, T x_{n}\right)}{2} \\
& =\frac{d\left(x_{n-1}, T x_{n}\right)}{2} \frac{d\left(x_{n-1}, T x_{n}\right)}{2} \\
& \leq \frac{d\left(x_{n-1}, x_{n+1}\right)}{2} \\
& \leq \frac{d\left(x_{n-1}, x_{n}\right)+d\left(x_{n}, x_{n+1}\right)}{2} \\
& \leq \max \left\{d\left(x_{n-1}, x_{n}\right), d\left(x_{n}, x_{n+1}\right)\right\}
\end{aligned}
$$

then $d\left(x_{n}, x_{n+1}\right) \leq \psi\left(d\left(x_{n-1}, x_{n}\right)\right)$ (other case implies that $\left.d\left(x_{n}, x_{n+1}\right)=0\right)$. Thus,

$$
d\left(x_{n}, x_{n+1}\right) \leq \psi\left(d\left(x_{n-1}, x_{n}\right)\right)
$$

for all $n \geq 1$. We claim that $\psi(0)=0$. If $\psi(0)>0$, then $\psi^{2}(0) \geq$ $\psi(0)>0$ because $\psi$ is nondecreasing. On the other hand, since $\psi(t)<t$ for all $t>0$, we have $\psi^{2}(0)<\psi(0)$ which is a contradiction. Hence, $\psi(0)=0$. Let $d_{n}=d\left(x_{n}, x_{n+1}\right)$ for all $n$. If there exists a natural number $n_{0}$ such that $d_{n_{0}}=0$, then it is easy to see that $d_{n}=0$ for all $n \geq n_{0}$, and so $\lim _{n \rightarrow \infty} d_{n}=0$. Now suppose that $d_{n} \neq 0$ for all $n$. In this case, we have $d_{n} \leq$ $\psi\left(d_{n-1}\right)<d_{n-1}$ for all $n$. Hence, $\left\{d_{n}\right\}$ is a decreasing sequence, and so there exists $d \geq 0$ such that $\lim _{n \rightarrow \infty} d_{n}=d$. If $d>$ 0 , then $d_{n}>0$ for all $n$, and so $d_{n} \leq \psi\left(d_{n-1}\right)<d_{n-1}$ for all $n$. Since $\psi$ is upper and semicontinuous, we obtain $d=$ $\lim _{n \rightarrow \infty} d_{n} \leq \lim _{n \rightarrow \infty} \psi\left(d_{n-1}\right) \leq \psi\left(\lim _{n \rightarrow \infty} d_{n-1}\right)=\psi(d)<$ $d$ which is a contradiction. Thus, $\lim _{n \rightarrow \infty} d_{n}=0$. Now, we prove that $\left\{x_{n}\right\}$ is a Cauchy sequence. If $\left\{x_{n}\right\}$ is not a Cauchy sequence, then there exist $\varepsilon>0$ and natural numbers $m_{k}, n_{k}$ such that $m_{k}>n_{k} \geq k$ and $d\left(x_{m_{k}}, x_{n_{k}}\right) \geq \varepsilon$ for all $k \geq 1$. Also, we choose $m_{k}$ as small as possible such that

$$
d\left(x_{m_{k}-1}, x_{n_{k}}\right)<\varepsilon
$$


Thus, $\varepsilon \leq d\left(x_{m_{k}}, x_{n_{k}}\right) \leq d\left(x_{m_{k}}, x_{m_{k}-1}\right)+d\left(x_{m_{k}-1}, x_{n_{k}}\right) \leq$ $d_{m_{k}-1}+\varepsilon$ for all $k$. Hence, $\lim _{k \rightarrow \infty} d\left(x_{m_{k}}, x_{n_{k}}\right)=\varepsilon$. Since $T$ has the property $(K)$, we obtain

$$
\begin{aligned}
d\left(x_{m_{k}}, x_{n_{k}}\right) \leq & d\left(x_{m_{k}}, x_{m_{k}+1}\right)+d\left(x_{m_{k}+1}, x_{n_{k}+1}\right) \\
& +d\left(x_{n_{k}+1}, x_{n_{k}}\right) \\
\leq & d_{m_{k}}+H\left(T x_{m_{k}}, T x_{n_{k}}\right)+d_{n_{k}} \\
\leq & d_{m_{k}}+\beta\left(T x_{m_{k}}, T x_{n_{k}}\right) \\
& \times H\left(T x_{m_{k}}, T x_{n_{k}}\right)+d_{n_{k}} \\
\leq & d_{m_{k}}+\psi\left(N\left(x_{m_{k}}, x_{n_{k}}\right)\right)+d_{n_{k}}
\end{aligned}
$$

for all $k$. Since $\lim _{k \rightarrow \infty} d\left(x_{m_{k}}, x_{n_{k}}\right)=\varepsilon, \lim _{k \rightarrow \infty} N\left(x_{m_{k}}\right.$, $\left.x_{n_{k}}\right)=\varepsilon$. In fact,

$$
\begin{aligned}
& d\left(x_{m_{k}}, x_{n_{k}}\right) \\
& \leq N\left(x_{m_{k}}, x_{n_{k}}\right) \\
& =\max \left\{d\left(x_{m_{k}}, x_{n_{k}}\right), d\left(x_{m_{k}}, T x_{m_{k}}\right), d\left(x_{n_{k}}, T x_{n_{k}}\right),\right. \\
& \left.\frac{d\left(x_{m_{k}}, T x_{n_{k}}\right)+d\left(x_{n_{k}}, T x_{m_{k}}\right)}{2}\right\} \\
& \leq \max \left\{d\left(x_{m_{k}}, x_{n_{k}}\right), d\left(x_{m_{k}}, x_{m_{k+1}}\right), d\left(x_{n_{k}}, x_{n_{k+1}}\right)\right. \text {, } \\
& \left.\frac{d\left(x_{m_{k}}, x_{n_{k+1}}\right)+d\left(x_{n_{k}}, x_{m_{k+1}}\right)}{2}\right\} \\
& \leq \max \left\{d\left(x_{m_{k}}, x_{n_{k}}\right), d\left(x_{m_{k}}, x_{m_{k+1}}\right), d\left(x_{n_{k}}, x_{n_{k+1}}\right)\right. \text {, } \\
& \left(d\left(x_{m_{k}}, x_{n_{k}}\right)+d\left(x_{n_{k}}, x_{n_{k+1}}\right)\right. \\
& \left.+d\left(x_{n_{k}}, x_{m_{k}}\right)+d\left(x_{m_{k}}, x_{m_{\mathrm{k}+1}}\right)\right) \\
& \left.\times(2)^{-1}\right\}
\end{aligned}
$$

and so $\varepsilon=\lim _{k \rightarrow+\infty} d\left(x_{m_{k}}, x_{n_{k}}\right) \leq \lim _{k \rightarrow \infty} N\left(x_{m_{k}}, x_{n_{k}}\right) \leq \varepsilon$. Since $\psi$ is upper semicontinuous, by using $(*)$ we obtain

$$
\begin{aligned}
\varepsilon & =\lim _{k \rightarrow \infty} d\left(x_{m_{k}}, x_{n_{k}}\right) \\
& \leq \lim _{k \rightarrow \infty} \psi\left(N\left(x_{m_{k}}, x_{n_{k}}\right)\right) \leq \psi(\varepsilon)<\varepsilon,
\end{aligned}
$$

which is a contradiction, and so $\left\{x_{n}\right\}$ is a Cauchy sequence. Choose $x^{*} \in X$ such that $x_{n} \rightarrow x^{*}$. Now, note that

$$
\begin{aligned}
H\left(\left\{x_{n}\right\}, T x_{n}\right) & =\max \left\{d\left(x_{n}, T x_{n}\right), \sup _{y \in T x_{n}} d\left(x_{n}, y\right)\right\} \\
& =H\left(T x_{n-1}, T x_{n}\right)
\end{aligned}
$$

for all $n$, and so

$$
\begin{aligned}
H\left(\left\{x_{n}\right\}, T x_{n}\right) & =H\left(T x_{n-1}, T x_{n}\right) \\
& \leq \beta\left(T x_{n-1}, T x_{n}\right) H\left(T x_{n-1}, T x_{n}\right) \\
& \leq \psi\left(N\left(x_{n-1}, x_{n}\right)\right) \\
& \leq \psi\left(d\left(x_{n-1}, x_{n}\right)\right) \leq d\left(x_{n-1}, x_{n}\right)
\end{aligned}
$$

for all $n$, and so $\lim _{n \rightarrow \infty} H\left(\left\{x_{n}\right\}, T x_{n}\right)=0$. Since $T$ has the property $(R)$, we obtain

$$
\begin{aligned}
H\left(\left\{x^{*}\right\}, T x^{*}\right) \leq & d\left(x^{*}, x_{n}\right) \\
& +H\left(\left\{x_{n}\right\}, T x_{n}\right)+H\left(T x_{n}, T x^{*}\right) \\
\leq & d\left(x^{*}, x_{n}\right)+H\left(\left\{x_{n}\right\}, T x_{n}\right) \\
& +\beta\left(T x_{n}, T x^{*}\right) H\left(T x_{n}, T x^{*}\right) \\
\leq & d\left(x^{*}, x_{n}\right)+H\left(\left\{x_{n}\right\}, T x_{n}\right) \\
& +\psi\left(N\left(x_{n}, x^{*}\right)\right)
\end{aligned}
$$

for all $n$. If $N\left(x_{n}, x^{*}\right)=d\left(x^{*}, T x^{*}\right)$, then we have

$$
\begin{aligned}
& H\left(\left\{x^{*}\right\}, T x^{*}\right) \\
& \quad \leq d\left(x^{*}, x_{n}\right)+H\left(\left\{x_{n}\right\}, T x_{n}\right)+\psi\left(H\left(\left\{x^{*}\right\}, T x^{*}\right)\right)
\end{aligned}
$$

for all $n$. This implies that $H\left(\left\{x^{*}\right\}, T x^{*}\right) \leq \psi\left(H\left(\left\{x^{*}\right\}, T x^{*}\right)\right)$, and so

$$
H\left(\left\{x^{*}\right\}, T x^{*}\right)=0 .
$$

If $N\left(x_{n}, x^{*}\right)=d\left(x_{n}, x^{*}\right)$ or $N\left(x_{n}, x^{*}\right) \leq d\left(x_{n}, x_{n+1}\right)$, then it is easy to see that $H\left(\left\{x^{*}\right\}, T x^{*}\right)=0$. Thus, $x^{*}$ is an endpoint of $T$.

Next example shows that a $\beta$-generalized weak contractive multifunction is not necessarily a generalized weak contractive multifunction.

Example 4. Let $X=\mathbb{R}$. Define $T: X \rightarrow C B(X)$ by $T x=[x, x+2]$ for all $x \in X$. Suppose that $\psi:[0,+\infty) \rightarrow$ $[0,+\infty)$ is an arbitrary upper semicontinuous function such that $\psi(t)<t$ for all $t>0$. If $x=0$ and $y=2$, then $H(T x, T y)=H([0,2],[2,4])=2$ and $N(x, y)=2$. Hence,

$$
H(T x, T y)=2 \gtreqless \psi(2)=\psi(N(x, y)) .
$$

Thus, $T$ is not a generalized weak contractive multifunction. Now, suppose that $\psi(t)=t / 2$ for all $t \geq 0$ and define $\beta$ : $2^{X} \times 2^{X} \rightarrow[0, \infty)$ by $\beta(A, B)=1 / 2$ for all subsets $A$ and $B$ of $X$. Then, we have

$$
\begin{aligned}
\beta(T x, T y) H(T x, T y) & =\frac{1}{2} d(x, y) \\
& =\psi(d(x, y))=\psi(N(x, y))
\end{aligned}
$$

for all $x, y \in \mathbb{R}$. Thus, $T$ is a $\beta$-generalized weak contractive multifunction. 
Next example shows that there are multifunctions which satisfy the conditions of Theorem 3, while they are not generalized weak contractive multifunctions.

Example 5. Let $X=[0,9 / 2]$ and let $d(x, y)=|x-y|$. Define $T: X \rightarrow C B(X)$ by

$$
T x= \begin{cases}\left\{\frac{x}{2}\right\} & 0 \leq x \leq 1 \\ \left\{4 x-\frac{3}{2}\right\} & 1<x \leq \frac{3}{2} \\ \{0\} & \frac{3}{2}<x \leq \frac{9}{2} .\end{cases}
$$

If $x=1$ and $y=3 / 2$, then

$$
\begin{aligned}
H(T x, T y) & =H\left(\left\{\frac{1}{2}\right\},\left\{\frac{9}{2}\right\}\right)=4>3 \\
& =N(x, y)>\psi(N(x, y)),
\end{aligned}
$$

where $\psi:[0,+\infty) \rightarrow[0,+\infty)$ is an arbitrary upper semicontinuous function such that $\psi(t)<t$ for all $t>0$. Thus, $T$ is not a generalized weak contractive multifunction. Now, we show that $T$ satisfies all conditions of Theorem 3. For this aim, define $\psi(t)=t / 2$ and $\beta(A, B)=1$ whenever $A$ and $B$ are subsets of $[0,1]$ and $\beta(A, B)=0$ otherwise. First suppose that $x \notin[0,1]$ or that $y \notin[0,1]$. If $x, y \in(3 / 2,9 / 2]$, then $T x, T y \subset[0,1]$ and $\beta(T x, T y)=1$. But, $H(T x, T y)=0$, and so $\beta(T x, T y) H(T x, T y) \leq \psi(N(x, y))$. If $x \in(1,3 / 2]$ or $y \in$ $(1,3 / 2]$, then $T x \nsubseteq[0,1]$, or $T y \nsubseteq[0,1]$ and so $\beta(T x, T y)=0$. Hence, $\beta(T x, T y) H(T x, T y) \leq \psi(N(x, y))$. Now, suppose that $x, y \in[0,1]$. In this case, we have $\beta(T x, T y) \geq$ $1, H(T x, T y)=H(\{x / 2\},\{y / 2\})=(1 / 2) d(x, y)$, and $N(x, y)=\max \{d(x, y), x / 2, y / 2,(d(x, y / 2)+d(y, x / 2)) / 2\}$. Thus, $d(x, y) \leq N(x, y)$, and so

$$
\begin{aligned}
\beta(T x, T y) H(T x, T y) & =\frac{1}{2} d(x, y) \\
& \leq \psi(d(x, y)) \leq \psi(N(x, y)) .
\end{aligned}
$$

Therefore, $T$ is a $\beta$-generalized weak contractive multifunction. Now, we show that $T$ is $\beta$-admissible. If $\beta(A, B) \geq 1$, then $A, B \subset[0,1]$, and so $T x=\{x / 2\} \in[0,1]$ and $T y=\{y / 2\} \in$ $[0,1]$ for all $x \in A$ and $y \in B$. Thus, $\beta(T x, T y) \geq 1$ for all $x \in A$ and $y \in B$. Now, suppose $A=[0,1 / 2]$ and $x_{0}=1 / 4$. Then, $T x_{0}=\{1 / 8\} \in[0,1]$ and $[0,1 / 2] \subset[0,1]$. Hence, $\beta\left(A, T x_{0}\right) \geq 1$. Now, we show that $T$ satisfies the condition $(H)$. First note that, for each $\varepsilon>0$, there exists $z \in X$ such that $\sup _{a \in T z} d(z, a)<\varepsilon$. Now, we show that for each $x \in X$ there exists $y \in T x$ such that $H(T x, T y)=\sup _{b \in T y} d(y, b)$. If $0 \leq x \leq 1$, then $T x=\{x / 2\}, T(x / 2)=\{x / 4\}$, and

$$
\begin{aligned}
H\left(T x, T\left(\frac{x}{2}\right)\right) & =H\left(\left\{\frac{x}{2}\right\},\left\{\frac{x}{4}\right\}\right) \\
& =\frac{x}{4}=\sup _{b \in T(x / 2)} d\left(\frac{x}{2}, b\right) .
\end{aligned}
$$

Since for $1<x \leq 3 / 2$ we have $5 / 2<4 x-(3 / 2) \leq 9 / 2$, $T(4 x-(3 / 2))=\{0\}$. Thus,

$$
\begin{aligned}
H\left(T x, T\left(4 x-\frac{3}{2}\right)\right) & =H\left(\left\{4 x-\frac{3}{2}\right\},\{0\}\right) \\
& =4 x-\frac{3}{2}=\sup _{b \in T(4 x-3 / 2)} d\left(4 x-\frac{3}{2}, b\right) .
\end{aligned}
$$

If $3 / 2<x \leq 9 / 2$, then $T x=\{0\}$ and $T(0)=\{0\}$. Hence,

$$
\begin{aligned}
H(T x, T(0)) & =H(\{0\},\{0\}) \\
& =0=\sup _{b \in T(0)} d(0, b) .
\end{aligned}
$$

It is easy to check that $T$ satisfies the conditions $(R)$ and $(K)$. Note that, 0 is the endpoint of $T$.

Now, we add an assumption to obtain uniqueness of endpoint. In this way, we introduce a new notion. Let $X$ be a set and $\beta: 2^{X} \times 2^{X} \rightarrow[0, \infty)$ a map. We say that the set $X$ has the property $\left(G_{\beta}\right)$ whenever $\beta(A, B) \geq 1$ for all subsets $A$ and $B$ of $X$ with $A \nsubseteq B$ or $B \nsubseteq A$.

Corollary 6. Let $(X, d)$ be a complete metric space, $\beta$ : $2^{X} \times 2^{X} \rightarrow[0, \infty)$ a mapping, and $T: X \rightarrow C B(X)$ a $\beta$-admissible, $\beta$-generalized weak contractive multifunction which has the properties $(R),(K)$, and $(H)$. Suppose that there exist a subset $A$ of $X$ and $x_{0} \in A$ such that $\beta\left(A, T x_{0}\right) \geq 1$. If $T$ has the approximate endpoint property and $X$ has the property $\left(G_{\beta}\right)$, then $T$ has a unique endpoint.

Proof. By using Theorem 3, $T$ has a endpoint. If $T$ has two distinct endpoints $x^{*}$ and $y^{*}$, then $\beta\left(T x^{*}, T y^{*}\right)=$ $\beta\left(\left\{x^{*}\right\},\left\{y^{*}\right\}\right) \geq 1$ because $X$ has the property $\left(G_{\beta}\right)$. Hence,

$$
\begin{aligned}
d\left(x^{*}, y^{*}\right) & \leq H\left(T x^{*}, T y^{*}\right) \\
& \leq \beta\left(T x^{*}, T y^{*}\right) H\left(T x^{*}, T y^{*}\right) \\
& \leq \psi\left(N\left(x^{*}, y^{*}\right)\right)<N(x *, y *) \\
& =d\left(x^{*}, y^{*}\right),
\end{aligned}
$$

which is a contradiction. Thus, $T$ has a unique endpoint.

In Example 5, $T$ has a unique endpoint, while $X$ does has not the property $\left(G_{\beta}\right)$. Also, $T$ has the property $(R)$, while $T$ is not lower semicontinuous. To see this, consider the sequence $\left\{x_{n}\right\}$ defined by

$$
x_{n}= \begin{cases}1-\frac{1}{n} & n=2 k \\ 1+\frac{1}{n} & n=2 k-1\end{cases}
$$

for $k \geq 1$ and put $y=1 / 2$ and $x_{0}=1$. Then $x_{n} \rightarrow 1$ and $y \in$ $T x_{0}=\{1 / 2\}$. Let $\left\{y_{n}\right\}$ be an arbitrary sequence in $X$ such that $y_{n} \in T x_{n}$ for all $n \geq 1$. Then, $y_{2 k-1} \in T x_{2 k-1}$ and $y_{2 k} \in T x_{2 k}$ for all $k$. But, $y_{2 k-1}=4 x_{2 k-1}-(3 / 2)$ for sufficiently large $k$ and $y_{2 k}=x_{2 k} / 2$ for all $k$ since $y_{2 k-1} \rightarrow 5 / 2, y_{n} \rightarrow 1 / 2$. This implies that $T$ is not lower semicontinuous. 
Corollary 7. Let $(X, d)$ be a complete metric space, $\beta: 2^{X} \times$ $2^{X} \rightarrow[0, \infty)$ a mapping, and $T: X \rightarrow C B(X)$ a $\beta$ admissible multifunction which has the properties $(R),(K)$, and $(H)$. Suppose that $X$ has the property $\left(G_{\beta}\right)$, and there exist a subset $A$ of $X, x_{0} \in A$ and $k \in[0,1)$ such that $\beta\left(A, T x_{0}\right) \geq 1$ and $\beta(T x, T y) H(T x, T y) \leq k N(x, y)$ for all $x, y \in X$. Then $T$ has a unique endpoint if and only if $T$ has the approximate endpoint property.

Proof. It is sufficient that we define $\psi(t)=k t$ for all $t \geq 0$. Then, Theorem 3 and Corollary 6 guarantee the result.

It has been proved that lower semicontinuity of the multifunction $T$ and the property $(R)$ are independent conditions [9]. We can replace lower semicontinuity of the multifunction instead of the property $(R)$ to obtain the next result. Its proof is similar to the proof of Theorem 3.

Theorem 8. Let $(X, d)$ be a complete metric space, $\beta: 2^{X} \times$ $2^{X} \rightarrow[0, \infty)$ a mapping, and $T: X \rightarrow C B(X)$ a lower semicontinuous, $\beta$-admissible, $\beta$-generalized weak contractive multifunction which has the properties $(K)$ and $(H)$. Suppose that there exist a subset $A$ of $X$ and $x_{0} \in A$ such that $\beta\left(A, T x_{0}\right) \geq 1$. Then $T$ has the approximate endpoint property if and only if $T$ has an endpoint.

Corollary 9. Let $(X, d)$ be a complete metric space, $\beta: 2^{X} \times$ $2^{X} \rightarrow[0, \infty)$ a mapping, and $T: X \rightarrow C B(X)$ a lower semicontinuous, $\beta$-admissible, $\beta$-generalized weak contractive multifunction which has the properties $(K)$ and $(H)$. Suppose that there exist a subset $A$ of $X$ and $x_{0} \in A$ such that $\beta\left(A, T x_{0}\right) \geq 1$. If $T$ has the approximate endpoint property and $X$ has the property $\left(G_{\beta}\right)$, then $T$ has a unique endpoint.

Corollary 10. Let $(X, d)$ be a complete metric space, $\beta: 2^{X} \times$ $2^{X} \rightarrow[0, \infty)$ a mapping, and $T: X \rightarrow C B(X)$ a $\beta-$ admissible multifunction which has the properties $(R),(K)$, and $(H)$. Suppose that $X$ has the property $\left(G_{\beta}\right)$, and there exist a subset $A$ of $X, x_{0} \in A$ and $k \in[0,1)$ such that $\beta\left(A, T x_{0}\right) \geq 1$ and $\beta(T x, T y) H(T x, T y) \leq k N(x, y)$ for all $x, y \in X$. If T has the approximate endpoint property, then $\operatorname{Fix}(T)=\operatorname{End}(T)=$ $\{x\}$.

Proof. If we put $\psi(t)=k t$, then, by using Theorem 2.10 in [9], $T$ has a fixed point. Since $T$ has the approximate endpoint property, by using Corollary 7, $T$ has a unique endpoint such $x$. Let $y \in \operatorname{Fix}(T)$. If $T x=T y$, then $y=x$. If $T x \neq T y$, then $\beta(T x, T y) \geq 1$ because $X$ has the property $\left(G_{\beta}\right)$. Also, we have

$$
\begin{aligned}
d(x, y) \leq H(\{x\}, T y) & =H(T x, T y) \\
& \leq \beta(T x, T y) H(T x, T y) \\
& \leq k N(x, y) .
\end{aligned}
$$

But, $N(x, y)=\max \{d(x, y), d(x, T x), d(y, T y),(d(x, T y)+$ $d(y, T x)) / 2\}=d(x, y)$. Thus, $d(x, y)=0$, and so $\operatorname{Fix}(T)=$ $\operatorname{End}(T)=\{x\}$.

Next corollary shows us the role of a point in the existence of endpoints.
Corollary 11. Let $(X, d)$ be a complete metric space, $x^{*} \in X a$ fixed element, and $T: X \rightarrow C B(X)$ a multifunction such that $T$ has the property $(H)$ and $x^{*} \in T x \cap T y$ for all subsets $A$ and $B$ of $X$ with $x^{*} \in A \cap B$ and all $x \in A$ and $y \in B$. Assume that $H(T x, T y) \leq \psi(N(x, y))$ for all $x, y \in X$ with $x^{*} \in T x \cap T y$, where $\psi:[0,+\infty) \rightarrow[0,+\infty)$ is a nondecreasing upper semicontinuous function such that $\psi(t)<t$ for all $t>0$. Suppose that there exist a subset $A_{0}$ of $X$ and $x_{0} \in A_{0}$ such that $x^{*} \in A_{0} \cap T x_{0}$. Assume that for each convergent sequence $\left\{x_{n}\right\}$ in $X$ with $x_{n} \rightarrow x$ and $x^{*} \in T x_{n-1} \cap T x_{n}$, for all $n \geq 1$, one has $x^{*} \in T x_{n} \cap T x$. Also, for each sequence $\left\{x_{n}\right\}$ in $X$ with $x^{*} \in T x_{n-1} \cap T x_{n}$ for all $n \geq 1$, there exists a natural number $k$ such that $x^{*} \in T x_{m} \cap T x_{n}$ for all $m>n \geq k$. Then $T$ has an endpoint if and only if $T$ has the approximate endpoint property.

Proof. It is sufficient we define $\beta: 2^{X} \times 2^{X} \rightarrow[0, \infty)$ by $\beta(A, B)=1$ whenever $x^{*} \in A \cap B$ and $\beta(A, B)=0$ otherwise, and then we use Theorem 3 .

Corollary 12. Let $(X, d)$ be a complete metric space, $x^{*} \in X$ a fixed element and $T: X \rightarrow C B(X)$ a lower semicontinuous multifunction such that $T$ has the property $(H)$ and $x^{*} \in T x \cap$ Ty for all subsets $A$ and $B$ of $X$ with $x^{*} \in A \cap B$ and all $x \in A$ and $y \in B$. Assume that

$$
H(T x, T y) \leq \psi(N(x, y))
$$

for all $x, y \in X$ with $x^{*} \in T x \cap T y$, where $\psi:[0,+\infty) \rightarrow$ $[0,+\infty)$ is a nondecreasing upper semicontinuous function such that $\psi(t)<t$ for all $t>0$. Suppose that there exist a subset $A_{0}$ of $X$ and $x_{0} \in A_{0}$ such that $x^{*} \in A_{0} \cap T x_{0}$. Assume that for each convergent sequence $\left\{x_{n}\right\}$ in $X$ with $x_{n} \rightarrow x$ and $x^{*} \in T x_{n-1} \cap T x_{n}$ for all $n \geq 1$, we have $x^{*} \in T x_{n} \cap T x$. Then $T$ has an endpoint if and only if $T$ has the approximate endpoint property.

Proof. It is sufficient to define $\beta: 2^{X} \times 2^{X} \rightarrow[0, \infty)$ by $\beta(A, B)=1$ whenever $x^{*} \in A \cap B$ and $\beta(A, B)=0$ otherwise, and then we use Theorem 8 .

Let $(X, d, \leq)$ be an ordered metric space. Define the order $\preceq$ on arbitrary subsets $A$ and $B$ of $X$ by $A \preceq B$ if and only if for each $a \in A$ there exists $b \in B$ such that $a \leq b$. It is easy to check that $(C B(X), \preceq)$ is a partially ordered set.

Theorem 13. Let $(X, d, \leq)$ be a complete ordered metric space and $T$ a closed and bounded valued multifunction on $X$ such that $T$ has the property $(H)$ and $T x \leq T y$ for all subsets $A$ and $B$ of $X$ with $A \leq B$ and all $x \in A$ and $y \in B$. Assume that $H(T x, T y) \leq \psi(N(x, y))$ for all $x, y \in X$ with $T x \leq T y$, where $\psi:[0,+\infty) \rightarrow[0,+\infty)$ is a nondecreasing upper semicontinuous function such that $\psi(t)<t$ for all $t>0$. Suppose that there exist a subset $A_{0}$ of $X$ and $x_{0} \in A_{0}$ such that $A_{0} \leq T x_{0}$. Assume that for each convergent sequence $\left\{x_{n}\right\}$ in $X$ with $x_{n} \rightarrow x$ and $T x_{n-1} \leq T x_{n}$, for all $n \geq 1$, one has $T x_{n} \leq T x$. Also, for each sequence $\left\{x_{n}\right\}$ in $X$ with $T x_{n-1} \preceq T x_{n}$ for all $n \geq 1$, there exists a natural number $k$ such that $T x_{m} \preceq T x_{n}$ for all $m>n \geq k$. Then $T$ has an endpoint if and only if $T$ has the approximate endpoint property. 
Proof. Define $\beta(A, B)=1$ whenever $A \preceq B$ and $\beta(A, B)=0$ otherwise, and then we use Theorem 3 .

Corollary 14. Let $(X, d, \leq)$ be a complete ordered metric space and $T$ a closed and bounded valued multifunction on $X$ such that $T$ has the property $(H)$ and $T x \preceq T y$ for all subsets $A$ and $B$ of $X$ with $A \preceq B$, all $x \in A$, and $y \in B$. Assume that $H(T x, T y) \leq \psi(N(x, y))$ for all $x, y \in X$ with $T x \preceq T y$, where $\psi:[0,+\infty) \rightarrow[0,+\infty)$ is a nondecreasing upper semicontinuous function such that $\psi(t)<t$ for all $t>0$. Suppose that there exist a subset $A_{0}$ of $X$ and $x_{0} \in A_{0}$ such that $A_{0} \preceq T x_{0}$. Assume that for each convergent sequence $\left\{x_{n}\right\}$ in $X$ with $x_{n} \rightarrow x$ and $T x_{n-1} \preceq T x_{n}$, for all $n \geq 1$, one has $T x_{n} \preceq T x$. Also, for each sequence $\left\{x_{n}\right\}$ in $X$ with $T x_{n-1} \preceq T x_{n}$ for all $n \geq 1$, there exists a natural number $k$ such that $T x_{m} \preceq T x_{n}$ for all $m>n \geq k$. If $T$ has the approximate endpoint property and $A \preceq B$ for all subsets $A$ and $B$ of $X$ with $A \nsubseteq B$ or $B \nsubseteq A$, then $T$ has a unique endpoint.

Proof. Define $\beta(A, B)=1$ whenever $A \preceq B$ and $\beta(A, B)=0$ otherwise, and then we use Corollary 6.

Let $(X, d)$ be a metric space and $T: X \rightarrow 2^{X}$ a multifunction. We say that $T$ is an $H S$-multifunction whenever for each $x \in X$ there exists $y \in T x$ such that $H(T x, T y)=\sup _{b \in T y} d(y, b)$. It is obvious that each HSmultifunction is an multifunction which has the property $(H)$. Thus, one can conclude similar results to above ones for $H S$-multifunctions. Here, we provide some ones. Although by considering $H S$-multifunction we restrict ourselves, we obtain strange results with respect to above ones. One can prove the following by reading exactly the proofs of similar above results.

Theorem 15. Let $(X, d)$ be a complete metric space, $\beta: 2^{X} \times$ $2^{X} \rightarrow[0, \infty)$ a mapping, and $T: X \rightarrow C B(X)$ a $\beta$ admissible, $\beta$-generalized weak contractive $H S$-multifunction which has the properties $(R)$ and $(K)$. Suppose that there exist a subset $A$ of $X$ and $x_{0} \in A$ such that $\beta\left(A, T x_{0}\right) \geq 1$. Then $T$ has an endpoint, and so $T$ has the approximate endpoint property.

Theorem 16. Let $(X, d)$ be a complete metric space, $\beta: 2^{X} \times$ $2^{X} \rightarrow[0, \infty)$ a mapping, and $T: X \rightarrow C B(X) a$ lower semicontinuous, $\beta$-admissible, and $\beta$-generalized weak contractive HS-multifunction which has the property $(K)$. Suppose that there exist a subset $A$ of $X$ and $x_{0} \in A$ such that $\beta\left(A, T x_{0}\right) \geq 1$. Then $T$ has an endpoint, and so $T$ has the approximate endpoint property.

The next result is a consequence of Theorem 15 .

Corollary 17. Let $(X, d)$ be a complete metric space, $x^{*} \in X$ a fixed element, and $T: X \rightarrow C B(X)$ an $H S$-multifunction such that $x^{*} \in T x \cap T y$ for all subsets $A$ and $B$ of $X$ with $x^{*} \in A \cap B$, all $x \in A$, and $y \in B$. Assume that $H(T x, T y) \leq$ $\psi(N(x, y))$ for all $x, y \in X$ with $x^{*} \in T x \cap T y$, where $\psi$ : $[0,+\infty) \rightarrow[0,+\infty)$ is a nondecreasing upper semicontinuous function such that $\psi(t)<t$ for all $t>0$. Suppose that there exist a subset $A_{0}$ of $X$ and $x_{0} \in A_{0}$ such that $x^{*} \in A_{0} \cap T x_{0}$. Assume that for each convergent sequence $\left\{x_{n}\right\}$ in $X$ with $x_{n} \rightarrow x$ and $x^{*} \in T x_{n-1} \cap T x_{n}$ for all $n \geq 1$ one has $x^{*} \in T x_{n} \cap T x$. Also, for each sequence $\left\{x_{n}\right\}$ in $X$ with $x^{*} \in T x_{n-1} \cap T x_{n}$ for all $n \geq 1$, there exists a natural number $k$ such that $x^{*} \in T x_{m} \cap T x_{n}$ for all $m>n \geq k$. Then $T$ has an endpoint, and so $T$ has the approximate endpoint property.

The next result is a consequence of Theorem 16 .

Corollary 18. Let $(X, d, \leq)$ be a complete ordered metric space and $T$ a closed and bounded valued lower semicontinuous HSmultifunction on $X$ such that $T x \preceq T y$ for all subsets $A$ and $B$ of $X$ with $A \preceq B$, all $x \in A$, and $y \in B$. Assume that $H(T x, T y) \leq \psi(N(x, y))$ for all $x, y \in X$ with $T x \preceq T y$, where $\psi:[0,+\infty) \rightarrow[0,+\infty)$ is a nondecreasing upper semicontinuous function such that $\psi(t)<t$ for all $t>0$. Suppose that there exist a subset $A_{0}$ of $X$ and $x_{0} \in A_{0}$ such that $A_{0} \preceq T x_{0}$. Assume that for each sequence $\left\{x_{n}\right\}$ in $X$ with $T x_{n-1} \preceq T x_{n}$ for all $n \geq 1$, there exists a natural number $k$ such that $T x_{m} \preceq T x_{n}$ for all $m>n \geq k$. Then $T$ has an endpoint, and so $T$ has the approximate endpoint property.

\section{Acknowledgments}

This work was completed while the second author (Dr. Gopal) was visiting the Azarbaijan University of Shahid Madani, Azarshahr, Tabriz, Iran, during the summer of 2012. He thanks Professor Sh. Rezapour and the University for their hospitality and support. The second author gratefully acknowledges the support from the CSIR, govternment of India, Grant no.-25(0215)/13/EMR-II.

\section{References}

[1] A. Amini-Harandi, "Endpoints of set-valued contractions in metric spaces," Nonlinear Analysis: Theory, Methods and Applications, vol. 72, no. 1, pp. 132-134, 2010.

[2] R. P. Agarwal, D. O'Regan, and D. R. Sahu, Fixed Point Theory for Lipschitzian-Type Mappings with Applications, Springer, New York, NY, USA, 2009.

[3] S. Moradi and F. Khojasteh, "Endpoints of multi-valued generalized weak contraction mappings," Nonlinear Analysis: Theory, Methods and Applications, vol. 74, no. 6, pp. 2170-2174, 2011.

[4] B. Samet, C. Vetro, and P. Vetro, "Fixed point theorems for $\alpha$ - $\psi$-contractive type mappings," Nonlinear Analysis: Theory, Methods and Applications, vol. 75, no. 4, pp. 2154-2165, 2012.

[5] D. Baleanu, H. Mohammadi, and Sh. Rezapour, "Some existence results on nonlinear fractional differential equations," Philosophical Transactions of the Royal Society, vol. 371, Article ID 20120144, 2013.

[6] M. Jleli and B. Samet, "Best proximity points for $\alpha-\psi$-proximal contractive type mappings and applications," Bulletin des Sciences Mathématiques, 2013.

[7] M. A. Miandaragh, M. Postolache, and Sh. Rezapour, "Some approximate fixed point results for generalized-contractive mappings ," To appear in. Scientific Bulletin-University Politehnica of Bucharest A.

[8] Sh. Rezapour and J. H. Asl, "A simple method for obtaining coupled fixed points of $\alpha$ - $\psi$-contractive type mappings," International Journal of Analysis, Article ID 438029, 7 pages, 2013. 
[9] H. Alikhani, V. Rakočević, Sh. Rezapour, and N. Shahzad, "Fixed points of proximinal valued $\beta$ - $\psi$-contractive multifunctions," To appear in. Journal of Nonlinear and Convex Analysis.

[10] H. Alikhani, Sh. Rezapour, and N. Shahzad, "Fixed points of a new type contractive mappings and multifunctions," To appear in. Filomat.

[11] S. M. A. Aleomraninejad, Sh. Rezapour, and N. Shahzad, "On fixed points of $\alpha$ - $\psi$-contractive multifunctions," Fixed Point Theory and Applications, vol. 2012, article 212, 8 pages, 2012.

[12] B. Mohammadi, Sh. Rezapour, and N. Shahzad, "Some results on fixed points of $\alpha$ - $\psi$-ciric generalized multifunctions," Fixed Point Theory and Applications, vol. 2013, article 24, 2013.

[13] S. M. A. Aleomraninejad, Sh. Rezapour, and N. Shahzad, "Some fixed point results on a metric space with a graph," Topology and Its Applications, vol. 159, no. 3, pp. 659-663, 2012.

[14] J. M. Borwein and A. S. Lewis, Convex Analysis and Nonlinear Optimization: Theory and Examples, Springer, New York, NY, USA, 2000 


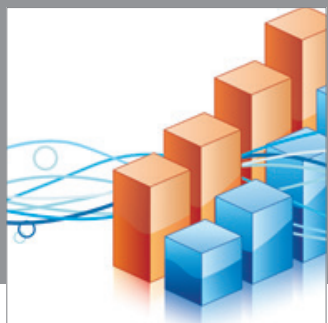

Advances in

Operations Research

mansans

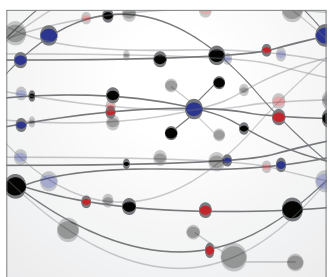

The Scientific World Journal
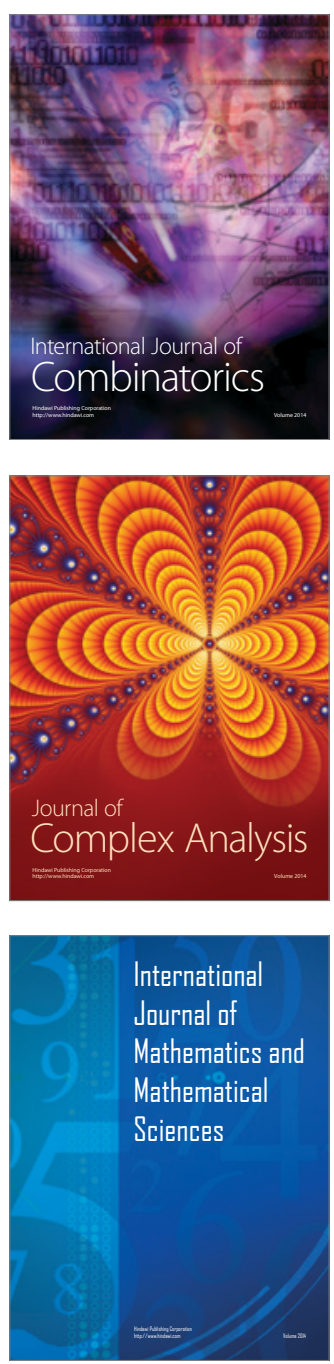
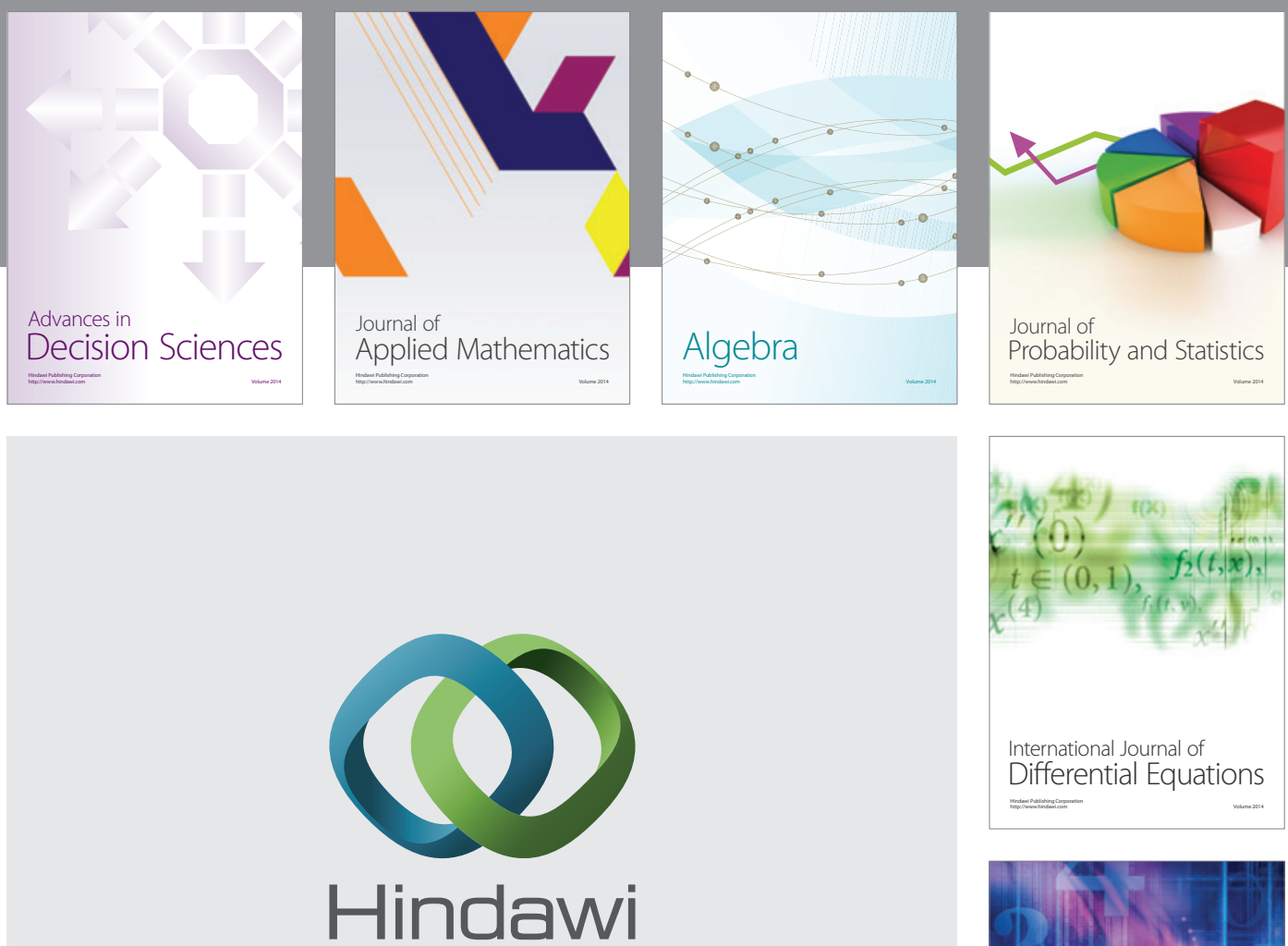

Submit your manuscripts at http://www.hindawi.com
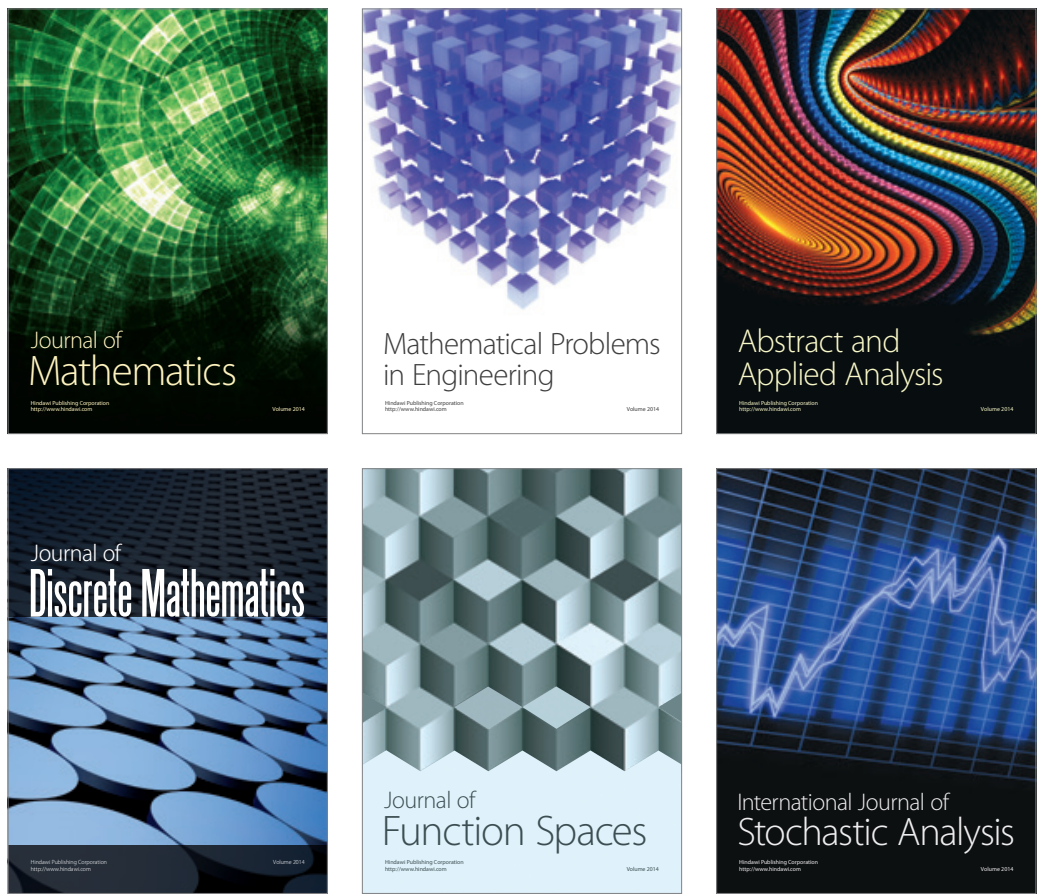

Journal of

Function Spaces

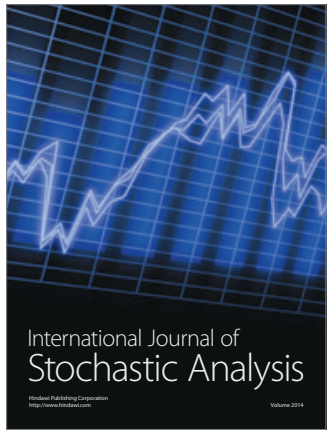

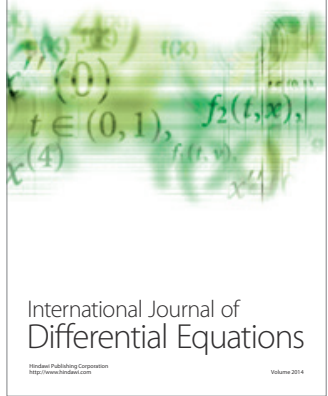
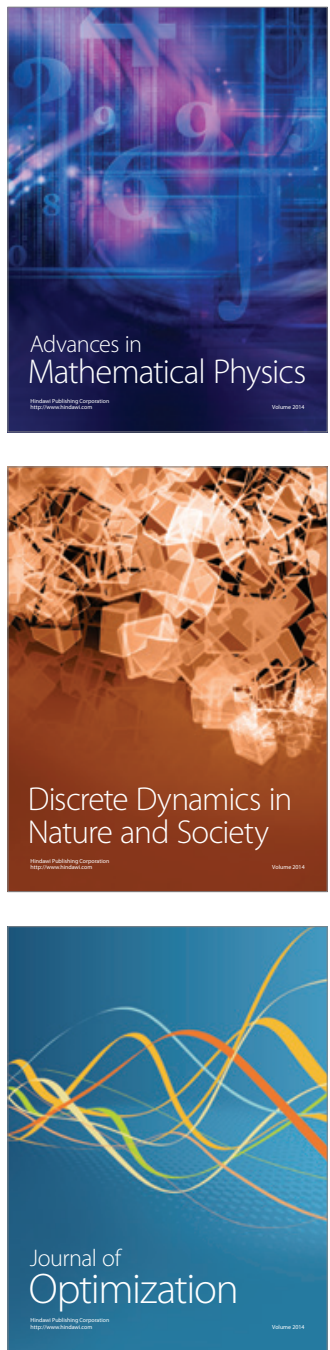